\title{
The Challenge of Evaluating Pain and a Pre-incisional Local Anesthetic Block
}

Background: Our objective was to test the effectiveness of a local anesthetic line block administered before surgery in reducing postoperative pain scores in dogs undergoing ovariohysterectomy (OVHX). Methods: This study is a prospective, randomized, blinded, clinical trial involving 59 healthy female dogs. An algometric pressure-measuring device was used to determine nociceptive threshold, and compared to three subjective pain scales. Group L/B received a line block of lidocaine $(4 \mathrm{mg} / \mathrm{kg}$ ) and bupivacaine $(1 \mathrm{mg} / \mathrm{kg})$ subcutaneously in the area of the incision site and saline subcutaneously as premedication; group L/BM (positive control) received a similar block and morphine $(0.5 \mathrm{mg} / \mathrm{kg})$ subcutaneously for premedication; and group SS (negative control) received a saline line block and saline premedication. Criteria for rescue analgesia were defined before the study. Dogs were assessed prior to surgery, at extubation (time 0) and at 2, 4, 6, 8 and 24 hours post-recovery. The data were analyzed with one-way ANOVA, and a Split Plot Repeated Measures ANOVA with one grouping factor and one repeat factor (time). $\mathrm{P}<0.05$ was considered statistically significant. Results: Approximately $33 \%$ of dogs required rescue analgesia at some point during the study, with no significant difference between groups. There was no significant difference between treatment groups with any assessment method. Conclusions: As there were no statistically significant differences between positive and negative controls, the outcome of this technique cannot be proven. 
1 Challenges in evaluation of pain and a pre-incisional line block

2 Carolyn M McKune ${ }^{\mathrm{a}}{ }^{*}$, Peter J Pascoe ${ }^{\mathrm{a}}$, B Duncan X Lascelles ${ }^{\mathrm{b}}$, Philip H Kass ${ }^{\mathrm{c}}$

$3 \quad{ }^{a}$ Department of Surgical and Radiological Sciences and

$4{ }^{\mathrm{c}}$ Department of Population Health and Reproduction, School of Veterinary Medicine,

5 University of California, Davis, CA, USA

6 bomparative Pain Research Laboratory, Department of Clinical Sciences \& Center for

7 Comparative Medicine and Translational Research, College of Veterinary Medicine, North

8 Carolina State University, Raleigh, NC, USA

9 †Current address: Mythos Veterinary LLC, Gainesville, FL, USA

$10{ }^{*}$ Corresponding author. tel.: +13522253636

11 Corresponding author's email address: mckune@mythosvet.com

12 Email addresses of all authors: mckune@mythosvet.com, pjpascoe@ucdavis.edu,

13 dxlascel@ncsu.edu, and phkass@ucdavis.edu 


\section{Abstract}

15 Background: Our objective was to test the effectiveness of a local anesthetic line block

16 administered before surgery in reducing postoperative pain scores in dogs undergoing 17 ovariohysterectomy (OVHX). This study was a prospective, randomized, blinded, clinical trial 18 involving 59 healthy female dogs. An algometric pressure-measuring device was used to 19 determine nociceptive threshold, and compared to three subjective pain scales. Group L/B 20 received a line block of lidocaine $(4 \mathrm{mg} / \mathrm{kg})$ and bupivacaine $(1 \mathrm{mg} / \mathrm{kg})$ subcutaneously in the 21 area of the proposed incision and saline subcutaneously as premedication; group L/BM (positive 22 control) received a similar block and morphine $(0.5 \mathrm{mg} / \mathrm{kg})$ subcutaneously for premedication;

23 and group SS (negative control) received a saline line block and saline premedication. Criteria for 24 rescue analgesia were defined before the study. Dogs were assessed prior to surgery, at extubation 25 (time 0 ) and at 2, 4, 6, 8 and 24 hours post-recovery. The data were analyzed with one-way 26 ANOVA, and a repeated measures ANOVA with one grouping factor and one repeat factor (time). $27 \mathrm{P}<0.05$ was considered statistically significant.

28 Results: Pain was so subtle that there were no significant differences between treatment groups

29 with any assessment method, and no significant difference between positive and negative 30 controls.

31 Conclusions: Pain in non-verbal responders is subtle, even in animals with a known stimulus that 32 results in pain. Pre-emptive, intraoperative and post-operative analgesia is necessary regardless of 33 pain score within the first 24 hours. None of the pain scales evaluated were sensitive enough to 34 determine pain in all animals in this study.

35 Key words: Dog, lidocaine, bupivacaine, local anesthetic, pain assessment 
Introduction

As any verbal responder who has experienced pain may attest to, pain decreases quality of

38 life (1). Therefore, pain management in patients experiencing pain is crucial for improving

39 quality of life. Pain management of non-verbal patients is uniquely challenging because the

40 ability to effectively diagnose and treat pain becomes very subjective. Pain assessment in non-

41 verbal species has been investigated along three principal lines: a) objective measures of

42 physiologic responses to experimental pain, b) subjective or semi-objective assessment of

43 behavior postoperatively, and c) quantitative measures of postoperative behavior and physiology.

44 While studies using objective physiological data (i.e. variables such as heart rate, respiratory rate

45 and blood pressure) are easy to perform and analyze statistically, there is minimal evidence that

46 these measures are reliable indicators of pain (2, 3). Most peer-reviewed research studies in

47 veterinary medicine use subjective or semi-objective assessments of postoperative pain or

48 sensitivity of an anatomical site to assess outcomes.

Algometers are devices used to quantitate pressure required to elicit a response from a

50 subject; this is termed "nociceptive threshold". Algometers provide a (partially) objective

51 measurement of incisional sensitivity. The "threshold" reading is numeric and objective, but the

52 factor determining the threshold (behavioral response) is subjective. Various mechanical

53 threshold devices are validated to assess somatosensory processing changes (4).

54 Multimodal analgesia is the combination of analgesic drugs with different methods of

55 action, with the goal of reducing or preventing nociceptive stimulation at multiple receptors and

56 pathways. In humans, multimodal analgesia has been shown to decrease post-operative morbidity

57 and mortality, improve quality of life and patient satisfaction, and decrease the associated costs to

58 hospitals and insurance companies (5). In addition to the general agreement of a clinical benefit

59 to this approach (6), there are also an increasing number of research studies in non-verbal species

60 supporting multimodal analgesia (7-9). One simple way to include multimodal analgesia is the 
61 incorporation of a local anesthetic to desensitize a specific region, in combination with systemic

62 analgesic administration.

63 This study was designed to assess the effect of pre-incisional administration of a

64 combination of local anesthetics on post-operative pain, measured by subjective and objective

65 pain scores after canine ovariohysterectomy (OVHX). We hypothesized that pre-incisional

66 infiltration of the incision area with local anesthetic agents (group L/B) would result in similar

67 post-surgical pain levels compared to animals receiving local anesthetic and an opioid (group

$68 \mathrm{~L} / \mathrm{BM}$ ), and decreased post-surgical pain compared to animals not receiving any pre-operative

69 analgesics (group SS).

70 Materials and methods

71 This study examined 59 healthy intact female dogs admitted to a local animal shelter

72 (Sacramento Society for Prevention of Cruelty to Animals [SPCA], Sacramento, CA, USA),

73 ranging in age from six months to eight years old with weights ranging from $3.4-35.5 \mathrm{~kg}$. A

74 physical examination was performed, and temperature, heart rate, and respiratory rate were

75 recorded prior to sedation for anesthesia and surgery. Each dog had a packed cell volume (PCV),

76 total protein (TP), and blood urea nitrogen (Azostick, Bayer Corporation, Elkhart, IN, USA)

77 checked prior to surgery. Please see Table 1 for a summary of baseline data. No dogs with

78 abnormal physiologic parameters, abnormal blood tests, evidence of a previous OVHX, or

79 requiring extension of the incision beyond the blocked area were used in this study. All protocols

80 were approved by the University of California, Davis, Institutional Animal Care and Use

81 Committee, as well as by administrative study reviewers at the Sacramento Society for

82 Prevention of Cruelty to Animals (SSPCA).

83 Anesthesia

84 Dogs were allocated into one of three groups using a computer generated randomized

85 block design. All three groups were sedated with acepromazine (Acepromazine maleate, Vedro, 
St. Joseph, MO, USA) $(0.03 \mathrm{mg} / \mathrm{kg}$, subcutaneously [SC]) administered prior to catheter

87 placement. An 18-22-gauge (depending on the animal's weight) over the needle IV catheter was

88 placed in a cephalic vein for drug and fluid administration. Anesthesia was induced with propofol

89 (Diprivan, AstraZeneca LP, Wilmington, DE, USA) to effect and maintained with isoflurane

90 (Isoflurane, Abbot Laboratories, North Chicago, IL, USA) in oxygen to effect. Lactated Ringer's

91 solution was administered at $10 \mathrm{~mL} / \mathrm{kg} /$ hour until recovery. Heart rate, respiratory rate, and

92 systolic blood pressure were monitored throughout the procedure.

93 Dogs in group L/B received a line block prior to surgery in the incision area, consisting of 4

$94 \mathrm{mg} / \mathrm{kg}$ lidocaine (Lidocaine, Hospira Inc., Lake Forest, IL, USA) and $1.0 \mathrm{mg} / \mathrm{kg}$ bupivacaine

95 (Bupivacaine, Hospira Inc., Lake Forest, IL, USA). These dogs also received $0.05 \mathrm{mg} / \mathrm{kg}$ of

96 saline SC at the same time as acepromazine administration. Group L/B were test subject dogs, to

97 compare to positive and negative control groups. Dogs in the group L/BM received a line block

98 prior to surgery, using $4.0 \mathrm{mg} / \mathrm{kg}$ lidocaine and $1.0 \mathrm{mg} / \mathrm{kg}$ bupivacaine. These dogs also received

$990.5 \mathrm{mg} / \mathrm{kg}$ of morphine (Morphine sulfate, Baxter Health Care Corporation, Deerfield, IL, USA)

$100 \mathrm{SC}$ at the same time as acepromazine administration. Group L/BM was the positive control group

101 (i.e. dogs anticipated to have minimal pain). Group SS was the negative control group (i.e. dog

102 anticipated to have pain). Dogs in group SS received $0.275 \mathrm{~mL} / \mathrm{kg}$ of normal saline prior to

103 surgery in the incisional area. These dogs also received $0.05 \mathrm{mg} / \mathrm{kg}$ of saline SC at the same time

104 as acepromazine administration. Because we anticipated animals with pain, criteria for rescue

105 analgesia were defined prior to the study's commencement and strictly adhered to. The line block

106 or saline (depending on the group) was administered after induction of anesthesia and initial

107 surgical preparation of the field, approximately five minutes prior to surgical incision.

108 Line block procedure

109 Appendix 1 shows the line block in schematic form. Local anesthetic or saline (depending 110 on the group) was infused with a 2.5 inch, 22 -gauge spinal needle in three separate lines to form 
111 an inverted double "L" administration site. One third of the volume of drug or saline was

112 administered at each site, as volume allowed. The level of the first line (Appendix 1, "1") was

113 roughly halfway between the umbilicus and the first set of nipples below the umbilicus;

114 placement was guided by consultation with the surgeon prior to incision to ensure coverage of the

115 area to be incised (Appendix 1, "A"). The width of this first line ran mediolaterally for

116 approximately $1.25 \mathrm{~cm}$ on either side of midline. The second line (Appendix 1, "2") began at the

117 left-most lateral point of the first line, and ran craniocaudally for the length of the spinal needle

118 on the left side of midline. The third line (Appendix 1, “3”) paralleled the second on the right side

119 of the umbilicus. In Appendix 1, "B" denotes the pubis. These blocks were administered in the

120 subcutaneous and fascial planes. Aspiration prior to administration of the block was performed to

121 ensure the drugs were not given intravenously.

122 Surgical procedure

123 The hair was clipped from the xiphoid process to the pubis and three cm laterally to the

124 nipple on both sides of the abdomen. The skin was scrubbed with chlorhexedine and rinsed with

125 water three times. The line block was applied after initial preparation; additional preparation

126 followed until the area was aseptically prepared. An incision was made extending below the

127 umbilicus to one-third the distance from the umbilicus to the pubis. An OVHX was performed in

128 a standard fashion (10) by one of three experienced, shelter veterinary surgeons. The skin was

129 closed in a routine manner.

130 Assessment

131 Four pain scoring assessments were used; initial values for each were recorded prior to

132 the sedation of the animal for anesthesia and surgery (time negative one). Assessments were then

133 made at zero (time of extubation), two, four, six, eight, and 24 hours postoperatively by one

134 veterinarian (CMM) who was blinded to which treatment group each animal was in. Caretakers 
135 made additional assessments during the day when animals were handled, to ensure any animal

136 that needed additional analgesia would receive it.

137 The first pain scoring assessment was a visual analog scale (VAS) score. This assessment

138 was made prior to any manipulation or handling of the animal. A mark on a ten centimeter $(\mathrm{cm})$

139 line corresponded to the assessor's visual assessment of the animal's pain, ranging from zero ("no

140 pain") to ten cm ("the most pain an animal could possibly be in"), measured in mm using a

141 standard ruler at each scoring assessment, and recorded after each measurement was taken.

142 The next two pain scoring assessments were done sequentially. One of these pain scales

143 was based on a previously validated scoring system, the Glasgow Composite Pain Scale (GCPS,

144 http://www.gla.ac.uk/faculties/vet/smallanimalhospital/ourservices/painmanagementandacupunct

145 ure, subheading: Short form pain questionnaire). The primary variables included vocalization

146 (quiet, crying, groaning, screaming), attention to painful area (ignoring, looking, licking, rubbing,

147 or chewing), mobility (normal, lame, slow or reluctant, stiff, or refusal to move), response to

148 touch (none, looking around, flinch, growl, snap, or cry), demeanor (happy and content, bouncy,

149 quiet, non-responsive or indifferent to surroundings, nervous or anxious or fearful, or depressed

150 or non-responsive to stimulation), and posture (comfortable, unsettled, restless, hunched or tense,

151 or rigid). Additional assessment was made using the University of Melbourne Pain Scale (UMPS)

152 (11). The primary variables included physiologic data (dilated pupils, percentage increase in heart

153 rate, percentage increase in respiratory rate, rectal temperature, salivation), response to palpation

154 (no change, guards/reacts when touched, guards/reacts before touched), activity (at rest [sleeping

155 or semiconscious, awake], eating, restless [pacing, getting up and down], or rolling/thrashing),

156 mental status (submissive, overtly friendly, wary, or aggressive), posture (guarding or protecting

157 affected area, recumbency, standing or sitting with head up, standing with head down, moving, or

158 abnormal body posture [prayer/hunched]), and vocalization (none, vocalizing when touched,

159 intermittent vocalization, or continuous vocalization). 
161 Electronic Von Frey Anesthesiometer, Woodland Hills, CA, USA) (12). The tip of the von Frey 162 apparatus was placed one $\mathrm{cm}$ adjacent to the center of the incision. It was pressed with a slow, 163 continuous pressure until a response was noted, with a maximal force of $1000 \mathrm{~g}$. A response was 164 considered an acknowledgement that the stimulus was noxious; this included behaviors such as 165 withdrawing from the stimulus, a cry, active head turn to the stimulus, attempt to bite, etc. This

166 measurement was repeated three times at five-minute intervals, and each value was recorded as

167 force in grams. The average value of these three readings was used in the data analysis. At each 168 time point, algometer measurements were also taken from the lateral thoracic wall in the same 169 manner. These measurements, as well as pre-sedation measurements, acted as controls for 170 analysis.

171 Rescue analgesia protocol one category of the GCPS, any animal with a pain score of 8 or greater on the GCPS or who did not improve over time as compared to pre-sedation GCPS score, any animal developing aggression, or a combination of these previous factors. Animal handlers at the SPCA also had the opportunity to declare an animal as being in pain, based on their observation, and these animals also received rescue analgesia. Administration of rescue analgesia and the reason for administration was recorded, and these animals were included in assessments; see "Blinding, exclusion criteria and statistical analysis". Any animal receiving rescue analgesia was reassessed

18130 minutes later to ensure efficacy of the rescue analgesia administration.

182 Blinding, exclusion criteria, and statistical analysis

183 The evaluator (CMM) was blinded to which dog was in which group (i.e. L/B, L/BM or 184 SS) as well as to whether a placebo or a study drug was contained in a particular group. The 
statistician who performed the data analysis remained blinded to which study drug was contained

186 in each group until the analyses were completed.

187 Initial power calculations were performed prior to commencing the study. An alpha error

188 level was set at 5\%. Standard deviation was set at 1.8 Glasgow Composite Pain Scale units (13).

189 A beta error level was set at 20\%. These calculations indicated the need for approximately 19

190 dogs in each group to find significant differences in our study populations, assuming a difference

191 of 2.6 on the Glasgow Composite Pain Scale as being significant (13). The groups were analyzed

192 for differences in age, weight, preoperative temperature, heart rate, respiratory rate, BUN,

$193 \mathrm{PCV} / \mathrm{TS}$, propofol dose $[\mathrm{mg} / \mathrm{kg}]$, and time negative one algometric values, by means of one-way

194 ANOVA. Normality of the errors was assessed by visual inspection of a histogram of the errors

195 and a normal probability plot. Errors were considered normal if the histogram was unimodal and

196 approximately symmetrical (14), and the normal probability plot was an upwardly sloping,

197 approximately straight line. Homogeneity of variance was tested by means of a studentized

198 residual vs. means plot. The response variable of treatment groups was analyzed by means of a

199 repeated measures ANOVA with one grouping factor and one repeat factor (time). Those dogs

200 receiving rescue analgesia were analyzed in a similar fashion in two separate analyses: within

201 their collective treatment group and as a separate subgroup. $\mathrm{P}<0.05$ was considered statistically

202 significant.

203 Results

204 There were 20, 19 and 20 dogs in Groups L/B, L/BM, and SS, respectively, for a total of

20559 dogs. Twenty of the 59 dogs initially enrolled, required rescue analgesia (seven, three and ten

206 dogs in groups L/B, L/BM, and SS, respectively, with no significant differences in the proportion

207 requiring rescue analgesia between groups). Of all the predetermined rescue analgesia criteria,

208 the only criteria triggering administration of rescue analgesia were animals that achieved a

209 maximum score in any one category (mobility: refusal to move) of the GCPS and animals 
210 developing aggression. The majority of the dogs requiring rescue analgesia required it at time 0

211 (extubation; 18 of 20 dogs) for refusal to move. All fifty-nine dogs were included in the analysis;

212 additional analysis of the separate subgroup of dogs who received rescue analgesia showed

213 similar results to the analysis of all 59 dogs, but the low numbers of dogs remaining in the groups

214 after removal of those requiring rescue analgesia brought into question the validity and precision

215 of the statistical analyses (therefore, data not shown).

216 VAS, GCPS, and UMPS analyses showed no significant difference in pain scores between

217 treatment groups, and there was a significant effect of time (i.e. a decrease in pain scores over

218 time; Figures 1, 2, and 3). Algometric values were compared to one of two controls. Regardless

219 of whether the value obtained at the wound was compared to the thoracic measurement obtained

220 at the same time or compared to the pre-incisional control reading (i.e. measurement at abdomen /

221 control measure), there was no significant difference in values obtained between treatment

222 groups, and there was a significant effect of time (i.e. a decrease in pain scores over time; Figures

2234 and 5).

224 Discussion

225 We chose three different groups to test the efficacy of our line block to improve

226 postoperative pain scores and algometric values. One group of animals (L/BM) was selected to

227 receive morphine premedication to serve as the positive control group (i.e. the group anticipated

228 to have the best analgesia). The group of animals that did not receive analgesia (SS) served as

229 the negative control (i.e. the group anticipated as having pain). The treatment group of interest,

$230 \mathrm{~L} / \mathrm{B}$, was evaluated in comparison to these positive and negative controls. The most profound

231 result of our study was the lack of statistically significant differences between our positive and

232 negative control at any given time point; that is, there was no statistically significant difference

233 between an animal that received no pre-emptive analgesia and an animal receiving a full mu

234 opioid receptor agonist to provide analgesia, using any of the assessment methods. This result 
235 was surprising, not only from the perspective of rendering the effects of treatment only

236 speculative, but also in the implications this possesses for investigators researching pain in non-

237 verbal species.

238 There are a number of potential reasons for the results obtained. Study design is critical

239 to successfully identifying targeted outcome. One potential reason no significant difference

240 between pain scores for any treatment group was evident was the number of dogs included in the

241 study, thus limiting statistical power of our study. Our initial sample size calculations potentially

242 hindered the study in two ways. Firstly, we applied sample size calculations meant for two groups

243 to three groups. In retrospect, in order to correctly calculate our initial sample size, we would

244 modify alpha $(\mathrm{P}=0.05)$, with three groups and the number of potential comparisons (3), and

245 therefore use an alpha value of 0.017 (0.05/3); this was not done. Secondly, our initial sample

246 size calculations used a difference in the GCPS of 2.6, based on previous work (13). This was

247 regarded as the minimum difference that would be clinically relevant. |The differences in pain

248 scores in our study were smaller than this (Figure 2) and while increasing the number of animals

249 treated may possibly have reached statistical significance it would still have had little relevance

250 for the clinician. Additionally, because we cannot account for Type II error, our statistical analysis

251 is not conclusive.

252 The other aspect of study design was the intent to maximize the potential for successful

253 pain identification, and thus the inclusion of one group that did not receive any preemptive

254 analgesic medication (negative control). This decision was not made lightly, and the criteria were

255 very strict for the use of rescue analgesia because of this. Even in light of this group that

256 intentionally included, albeit aggressively managed for, pain, there was still no significant

257 difference between the negative and positive control groups.

258 It may be that the dogs in this study were experiencing little discomfort, making it

259 difficult to distinguish between the treatment groups. While this may seem unreasonable in 
regards to an intra-abdominal procedure, pain scores on the only validated scoring system

261 (GCPS) were very low, never achieving a score of greater than five out of a maximal value of 24

262 at any one time point. A study evaluating intervention levels using the GCPS suggested

263 intervening if a score of greater than seven out of 24 was obtained; the GCPSs values obtained in

264 the present study were below this threshold (15). With such low pain scores, it was difficult to

265 establish differences between the treatment groups. The low pain scores may have been due to the

266 highly experienced veterinarians who were performing the OVHX creating minimal tissue trauma

267 during surgery (and thus minimal pain associated with the surgery). In this study, the three

268 surgeons were shelter veterinarians who performed up to 40 surgeries on any given day with over

26930 years of combined experience between them; surgery time ranged from 11 to 47 minutes, with

270 an average surgery time of 21 minutes. This is considerably less than the average time of 140

271 minutes for a veterinary student to spay a dog (16). If a group of less experienced surgeons - for

272 example, veterinary student surgeons - performed the procedures, more detectable differences

273 may have arisen. There is extensive debate about this subject, further complicated by a lack of

274 reporting surgeon experience level in well-performed pain studies. At least one study specifically

275 examining surgeon experience level suggested experience level of the surgeon was not correlated

276 with a change in postoperative pain score (17). However, recent basic science evidence

277 underscores the importance of deep tissue trauma to the experience of pain (18). Basic science

278 work also supports this on a receptor level: surgical tissue injuries enhanced the membrane

279 translocation of receptors important in post-operative hypersensitivity $(19,20)$. Surgery

280 performed by experienced surgeons, as was the case in this study, may reduce post-operative pain

$281(21,22)$ to levels below the sensitivity of current pain assessment scales.

282 Another reason for low pain scores on various scales may be due to inherent insensitivity

283 of the measurement techniques, preventing a significant difference between positive and negative

284 controls. Surprisingly little work has been performed to produce validated assessment systems for 
acute pain, with the Glasgow Composite Pain Scale standing out as the most validated scale in

286 this regard (13). However, this scoring system was validated using a variety of surgical

287 procedures, including orthopedic procedures. Additionally, the GCPS has not undergone criterion

288 validation testing. It is possible that a dog undergoing OVHX by an experienced veterinarian may

289 have signs of pain more subtle than this assessment instrument can detect. The von Frey

290 apparatus was sensitive to changes in threshold testing with dogs given $1 \mathrm{mg} / \mathrm{kg}$ morphine (12),

291 and appears reliable in clinically normal dogs (4). However, data gathered by one of the authors

292 (BDXL) found no difference in von Frey thresholds when it was used to assess wounds being

293 infused with saline or with local anesthetic (23). This suggests that the von Frey may not be the

294 appropriate instrument for assessing sensitivity of clinical wounds. Testing site could make a

295 difference in the reliability of the algometer, as previous reports suggest that the canine carpal

296 pad may be the most satisfactory site for testing $(12,24)$. Because this location was considered

297 unusual for testing sensitivity of an abdominal wound, it was not used for either the control or the

298 test site, which may contribute to the difficulty of using the algometer for assessment. This topic

299 needs further research to understand why the results appear counterintuitive, and to understand

300 appropriate means to assess wound sensitivity.

301 There is no doubt that expertise of the assessor in regards to pain assessment plays a

302 major role, as evidenced by a single experienced anesthesiologist finding a statistically significant

303 improvement after an incisional block with bupivacaine in dogs undergoing a celiotomy (25). As

304 involved as veterinarians are in the care of animals on a daily basis, it is still possible to

305 misclassify an animal as not in pain for many reasons - including temperament, breed, type of

306 surgery, and surgeon experience. In a study comparing staff observations versus a self-report of

307 pain in young children, staff observations of pain were generally lower than the self-reports (26).

308 However, for animals there is little alternative to an observer for pain assessment. The negative

309 aspects of such a misclassification are obvious. The inclusion of multiple pain assessment tools 
with very defined criteria was intended to counter potential inexperience, but cannot negate the

311 possibility altogether. Although the differences in the three reduced-size groups that received

312 rescue analgesia failed to reach statistical significance, the difference between the L/BM group

313 and the SS group (16\% vs. 50\% treated), if real, is clinically important and suggests that the

314 clinical judgment of when to administer rescue analgesia includes factors that are not captured in

315 the scoring systems that were used. We elected to give rescue analgesia to any patient with a

316 maximum value in any one GCPS category (27-29), as a means to favor generous administration

317 of rescue analgesia for any patient who might need it. Our decision to give rescue analgesia to

318 patients with a maximum value in any one GCPS category may have biased our results, as 18 of

31920 dogs received rescue analgesia for a maximum value in the category of refusing to move post-

320 surgery. However, given the large number of patients in group SS that received rescue analgesia

321 (almost half of the animals in that group), it is possible that refusal to move may be a sensitive

322 indicator of patient discomfort in the patient with pain secondary to an OVHX.

The effect of time present (i.e. a decrease in pain scores over time) in this study suggests

324 that we do see changes in pain scale scores and von Frey readings over the course of a 24-hour

325 period. Using subjective pain scores, all values returned to baseline or near baseline by 24 hours,

326 suggesting that we could no longer detect pain effectively at that point. When assessing

327 algometric scores, there was an initial decrease from baseline after extubation, and while values

328 tended to move back towards baseline between eight and 24 hours, the values never returned to

329 baseline. This suggests wound sensitivity may still be present when subjective assessments do not

330 detect pain. An alternative explanation is that the dogs had become behaviorally sensitized to the

331 testing device. Ideally, testing of dogs that were not operated on would have been performed to

332 evaluate the effect of time on threshold readings. Data (30) suggest there is a learned response

333 that decreases thresholds over time in normal dogs, but the data were generated using a more

334 blunt device than the von Frey used in the present study. 
No adverse events were documented in this study to suggest that a local anesthetic

336 infiltrative block is harmful to a patient, as opposed to another study examining incisional line

337 block (31). Fitzpatrick et al. (2010) may have seen greater complications because they choose to

338 infiltrate the site of the incision, where as we infiltrated the tissue surrounding the incision. The

339 block took a short time ( $<2$ minutes) to perform. Other studies have found that incisional blocks

340 provide effective analgesia $(25,32)$.

\section{Conclusions}

342 We believe we cannot make firm conclusions about whether or not this line block is

343 effective due to the lack of statistically significant differences between positive and negative

344 controls. Indeed, the ability to assess pain in non-verbal species even with multiple assessment

345 tools is called into question with the results of this study, necessitating a humble and

346 compassionate approach to pain management in all non-verbal species.

347 The veterinary medical profession must work towards developing more sensitive and

348 specific assessments of pain to evaluate the effectiveness of postulated analgesic interventions,

349 while continuing to provide conscientious therapy knowing such strategies have not yet been

350 developed. If an experienced observer cannot detect a patient with known pain from one that

351 received adequate analgesia using four different techniques, it is relatively easy to miss a patient

352 experiencing pain that cannot self-communicate. If one is inducing something that is likely to

353 result in pain, aggressive pain management is warranted as a moral and ethical obligation. 
354 Appendix 1: Site for line block/infiltration of local anesthetic or saline. Please see text for 355 description of labels.

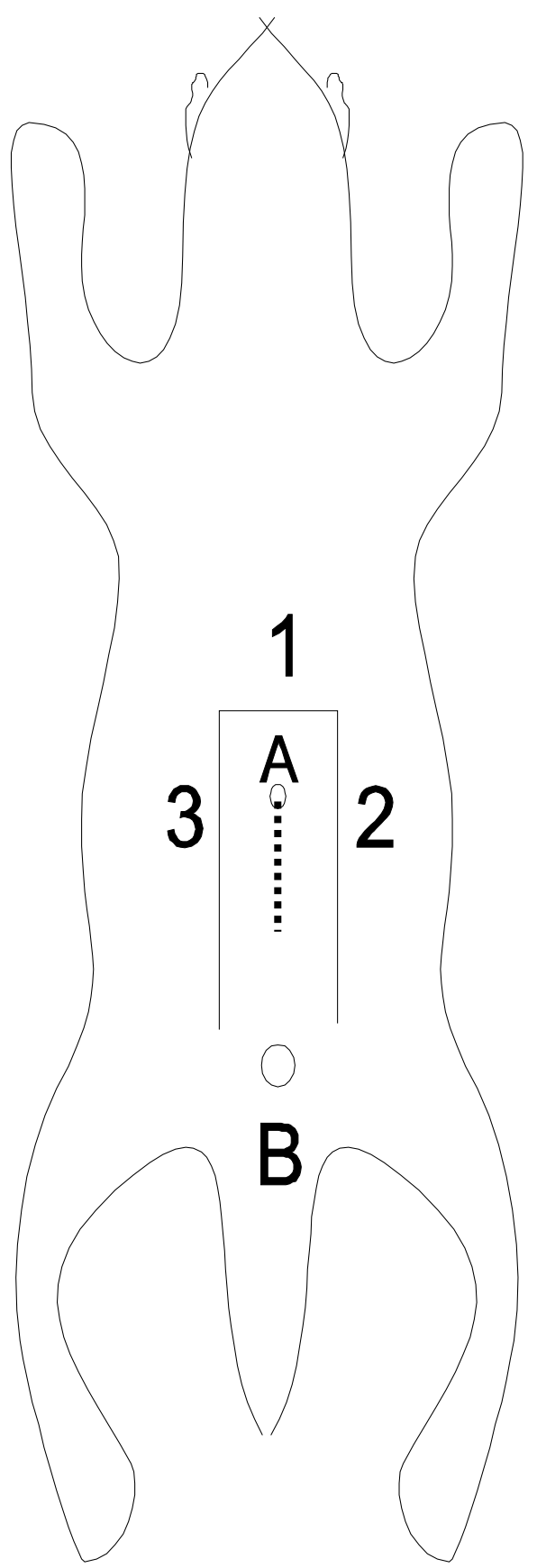


1. Niv D, Kreitler S. Pain and quality of life. Pain Pract. 2001;1(2):150-61.

357 2. Cambridge AJ, Tobias KM, Newberry RC, Sarkar DK. Subjective and objective

358 measurements of postoperative pain in cats. J Am Vet Med Assoc. 2000;217(5):685-90.

3593 3. Conzemius MG, Brockman DJ, King LG, Perkowski SZ. Analgesia in dogs after

360 intercostal thoracotomy: a clinical trial comparing intravenous buprenorphine and interpleural

361 bupivacaine. Vet Surg. 1994;23(4):291-8.

362 4. Briley JD, Williams MD, Freire M, Griffith EH, Lascelles BD. Feasibility and

363 repeatability of cold and mechanical quantitative sensory testing in normal dogs. Vet j. 2013.

364 5. Skinner HB. Multimodal acute pain management. Am J Orthop (Belle Mead, NJ.

365 2004;33(5 Suppl):5-9.

366 6. Lascelles BD, Main DC. Surgical trauma and chronically painful conditions--within our comfort level but beyond theirs? J Am Vet Med Assoc. 2002;221(2):215-22.

3687 Lascelles BD, Gaynor JS, Smith ES, Roe SC, Marcellin-Little D, Davidson G, et al.

369 Amantadine in a multimodal analgesic regimen for alleviation of refractory osteoarthritis pain in 370 dogs. J Vet Intern Med. 2008;22(1):53-9.

3718 8. Slingsby LS, Murrell JC, Taylor PM. Combination of dexmedetomidine with buprenorphine enhances the antinociceptive effect to a thermal stimulus in the cat compared with either agent alone. Vet Anaesth Analg. 2010;37(2):162-70.

9. Martins TL, Kahvegian MA, Noel-Morgan J, Leon-Román MA, Otsuki DA, Fantoni DT. Comparison of the effects of tramadol, codeine, and ketoprofen alone or in combination on postoperative pain and on concentrations of blood glucose, serum cortisol, and serum interleukin6 in dogs undergoing maxillectomy or mandibulectomy. Am J Vet Res. 2010;71(9):1019-26.

10. Fossum TW. Small Animal Surgery. 3rd Edition ed: Mosby; 2007. 1632 p.

11. Firth A, Haldane S. Development of a scale to evaluate postoperative pain in dogs. J Am Vet Med Assoc. 1999;214(5):651-9.

12. KuKanich B, Lascelles BD, Papich MG. Assessment of a von Frey device for evaluation of the antinociceptive effects of morphine and its application in pharmacodynamic modeling of morphine in dogs. Am J Vet Res. 2005;66(9):1616-22.

13. Morton CM, Reid J, Scott EM, Holton LL, Nolan AM. Application of a scaling model to establish and validate an interval level pain scale for assessment of acute pain in dogs. Am J Vet Res. 2005;66(12):2154-66.

14. Petrie A, Watson P. Statistics for Veterinary and Animal Science. Second ed. Oxford, UK: Blackwell Sciences; 2006.

15. Reid J, Nolan A, Hughes J, Lascelles B, Pawson P, Scott E. Development of the shortform Glasgow Composite Measure Pain Scale (GCMP-SF) and derivation of an analgesic intervention score. Animal Welfare. 2007(16(s)):97-104.

16. Kennedy KC, Tamburello KR, Hardie RJ. Peri-operative morbidity associated with ovariohysterectomy performed as part of a third-year veterinary surgical-training program. J Vet Med Educ. 2011;38(4):408-13.

17. Wagner AE, Worland GA, Glawe JC, Hellyer PW. Multicenter, randomized controlled trial of pain-related behaviors following routine neutering in dogs. J Am Vet Med Assoc. 2008;233(1):109-15.

18. Xu J, Brennan TJ. Guarding pain and spontaneous activity of nociceptors after skin versus skin plus deep tissue incision. Anesthesiology. 2010;112(1):153-64.

19. Wang Y, Wu J, Guo R, Zhao Y, Wang Y, Zhang M, et al. Surgical incision induces phosphorylation of AMPA receptor GluR1 subunits at Serine-831 sites and GluR1 trafficking in spinal cord dorsal horn via a protein kinase Cgamma-dependent mechanism. Neuroscience. 2013;240:361-70. 
404

405

406

407

408

409

410

411

412

413

414

415

416

417

418

419

420

421

422

423

424

425

426

427

428

429

430

431

432

433

434

435

436

437

438

439

440

441

442

443

20. Michelsen J, Heller J, Wills F, Noble GK. Effect of surgeon experience on postoperative plasma cortisol and C-reactive protein concentrations after ovariohysterectomy in the dog: a randomised trial. Aust Vet J. 2012;90(12):474-8.

21. Devitt CM, Cox RE, Hailey JJ. Duration, complications, stress, and pain of open ovariohysterectomy versus a simple method of laparoscopic-assisted ovariohysterectomy in dogs. J Am Vet Med Assoc. 2005;227(6):921-7.

22. Freeman LJ, Rahmani EY, Al-Haddad M, Sherman S, Chiorean MV, Selzer DJ, et al. Comparison of pain and postoperative stress in dogs undergoing natural orifice transluminal endoscopic surgery, laparoscopic, and open oophorectomy. Gastrointest Endosc. 2010;72(2):37380.

23. Hardie EM, Lascelles BD, Meuten T, Davidson GS, Papich MG, Hansen BD. Evaluation of intermittent infusion of bupivacaine into surgical wounds of dogs postoperatively. Vet J. 2011;190(2):287-9.

24. KuKanich B, Lascelles BD, Papich MG. Use of a von Frey device for evaluation of pharmacokinetics and pharmacodynamics of morphine after intravenous administration as an infusion or multiple doses in dogs. Am J Vet Res. 2005;66(11):1968-74.

25. Savvas I, Papazoglou LG, Kazakos G, Anagnostou T, Tsioli V, Raptopoulos D. Incisional block with bupivacaine for analgesia after celiotomy in dogs. J Am Anim Hosp Assoc. 2008;44(2):60-6.

26. Shavit I, Kofman M, Leder M, Hod T, Kozer E. Observational pain assessment versus self-report in paediatric triage. Emerg Med J. 2008;25(9):552-5.

27. Ahn J, Jeong M, Lee E, Kim S, Park S, Kang S, et al. Effects of peribulbar anesthesia (sub-Tenon injection of a local anesthetic) on akinesia of extraocular muscles, mydriasis, and intraoperative and postoperative analgesia in dogs undergoing phacoemulsification. Am J Vet Res. 2013;74(8):1126-32.

28. Tsai TY, Chang SK, Chou PY, Yeh LS. Comparison of postoperative effects between lidocaine infusion, meloxicam, and their combination in dogs undergoing ovariohysterectomy. Vet Anaesth Analg. 2013.

29. O O, Smith LJ. A comparison of epidural analgesia provided by bupivacaine alone, bupivacaine + morphine, or bupivacaine + dexmedetomidine for pelvic orthopedic surgery in dogs. Vet Anaesth Analg. 2013;40(5):527-36.

30. Coleman K, Schmeidt C, Kirkby K, Erickson A, Lascelles B, editors. Confounding Factors in Algometric Assessment of Mechanical Thresholds in Nor- mal Dogs. American College of Veterinary Surgeons Veterinary Symposium; 2011; Chicago, Il.

31. Fitzpatrick CL, Weir HL, Monnet E. Effects of infiltration of the incision site with bupivacaine on postoperative pain and incisional healing in dogs undergoing ovariohysterectomy. J Am Vet Med Assoc. 2010;237(4):395-401.

32. Carpenter RE, Wilson DV, Evans AT. Evaluation of intraperitoneal and incisional lidocaine or bupivacaine for analgesia following ovariohysterectomy in the dog. Vet Anaesth Analg. 2004;31(1):46-52. 


\section{Figure 1}

Figure 1

Visual Analogue Scale (VAS), from 0-10 centimeters, prior to premedication (time -1), extubation (time 0), and 2, 4, 6, 8 and 24 hours post-operatively. Note: L/B received saline premedication and local anesthetic line block, L/BM received morphine premedication and a local anesthetic line block, and SS received a saline premedication and saline line block. Error bars represent standard deviation.

圈 L/B

$\mathbf{v L / B M}$

घSS

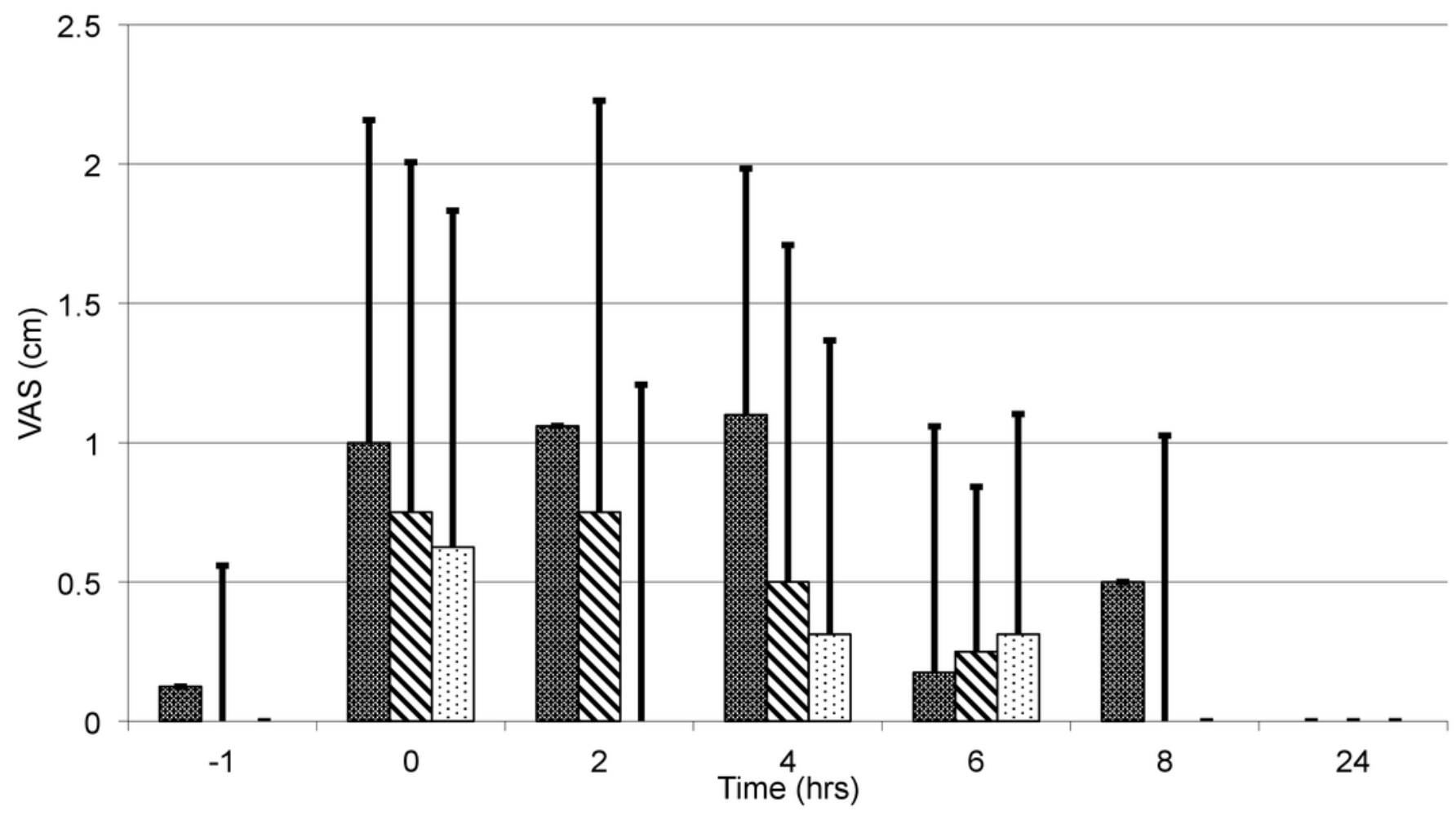




\section{Figure 2}

Figure 2

Glasgow composite pain scale (GCPS) scores from 0 to 24 prior to premedication (time -1), at extubation (time 0), and 2, 4, 6, 8 and 24 hours post-operatively. Note: L/B received saline premedication and local anesthetic line block, L/BM received morphine premedication and a local anesthetic line block, and SS received a saline premedication and saline line block. Error bars represent standard deviation.

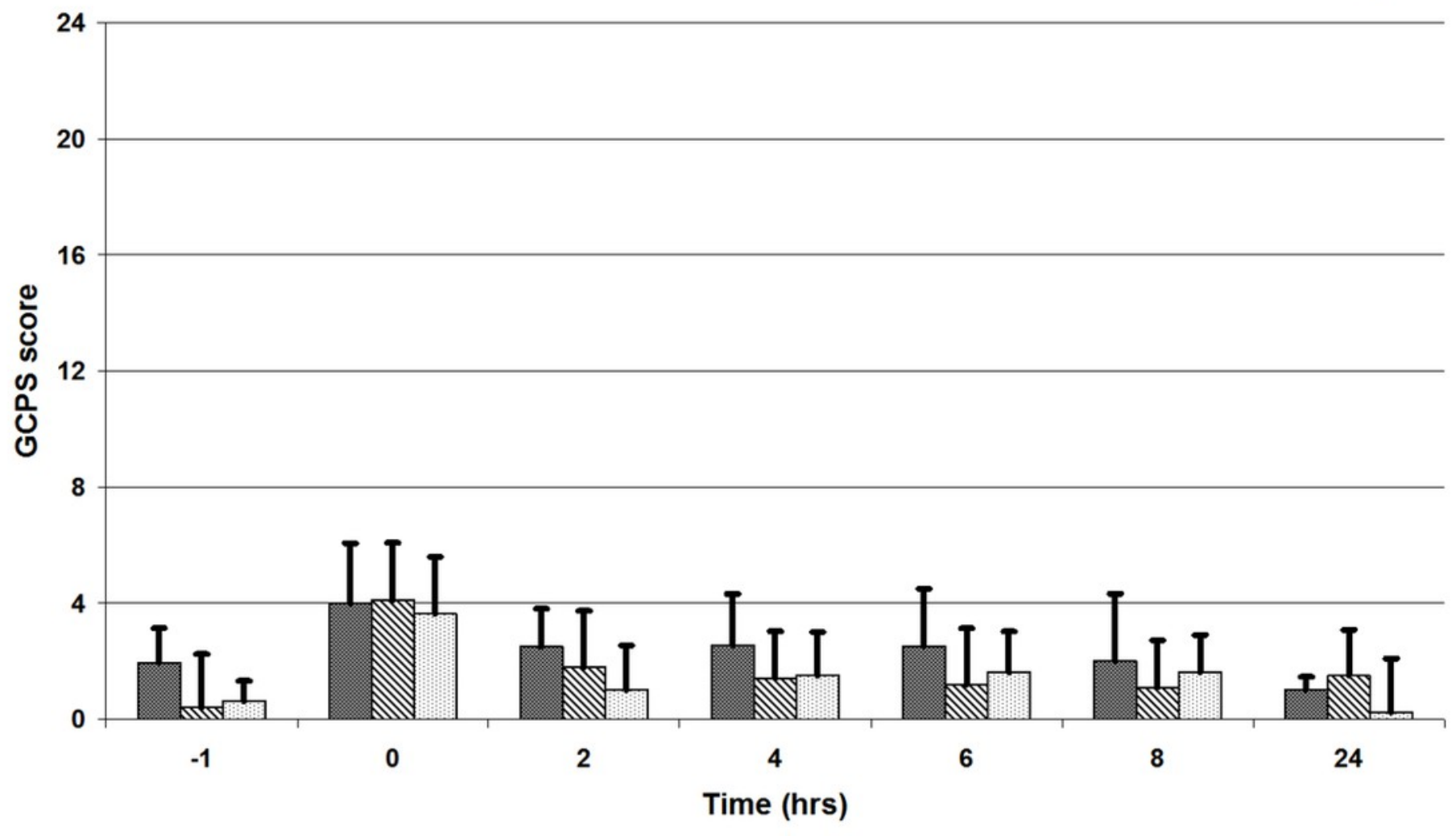




\section{Figure 3}

Figure 3

University of Melbourne Pain Scale scores from 0 to 27 prior to premedication (time -1 ), at extubation (time 0 ), and 2, 4, 6, 8 and 24 hours post-operatively. Note: L/B received saline premedication and local anesthetic line block, L/BM received morphine premedication and a local anesthetic line block, and SS received a saline premedication and saline line block. Error bars represent standard deviation.

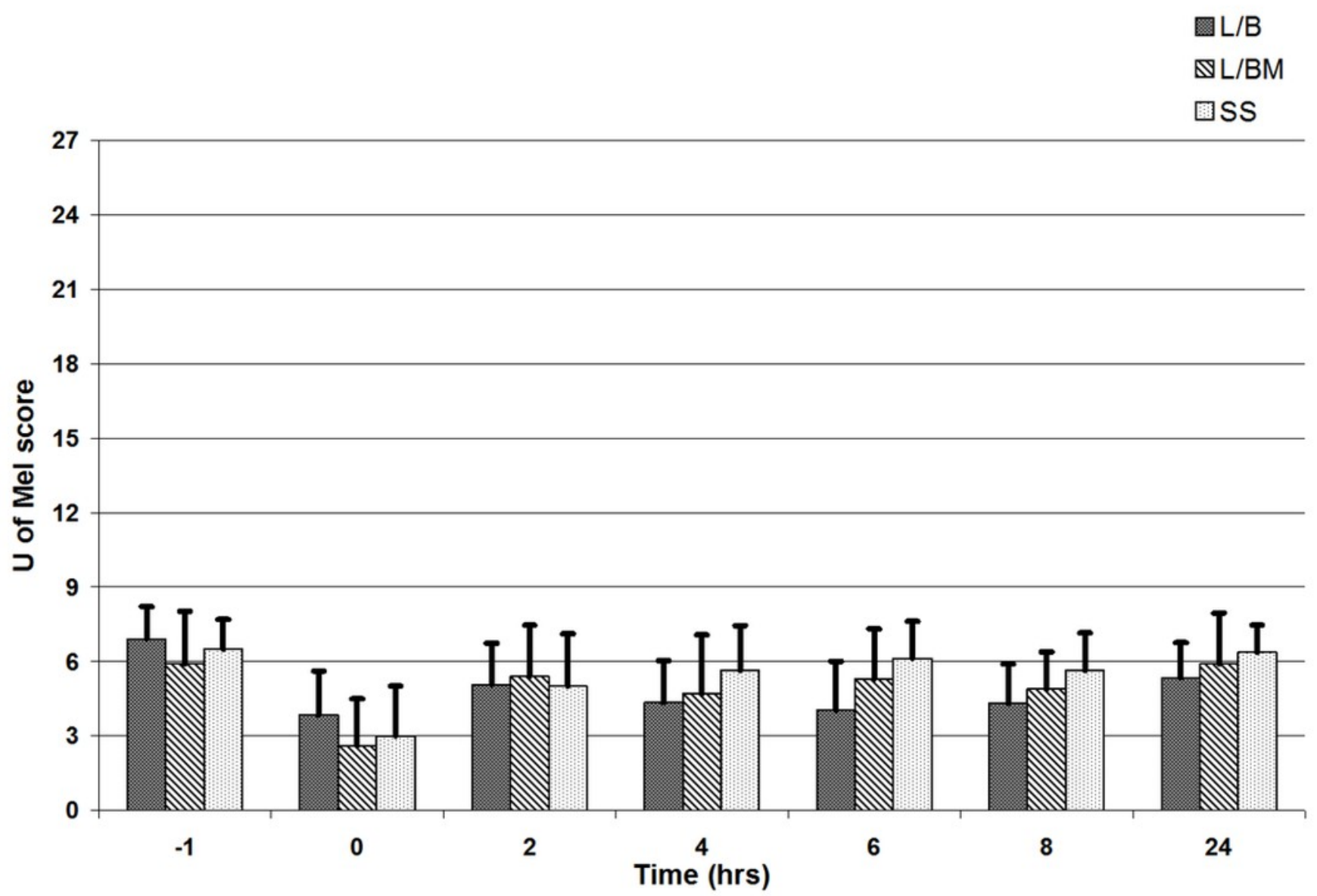




\section{Figure 4}

Figure 4

Algometric value, depicted as a ratio compared to the value obtained at the abdomen versus the value obtained at the thorax at the same time points: at premedication (time -1), at extubation (time 0), and at 2, 4, 6, 8 and 24 hours post-operatively. Note: L/B received saline premedication and local anesthetic line block, L/BM received morphine premedication and a local anesthetic line block, and SS received a saline premedication and saline line block. Also note that a ratio of one indicates the animal tolerates the same level of pressure on the abdomen as the thorax. A decreasing ratio indicates the animal tolerates less pressure on the abdomen as compared to the thorax.

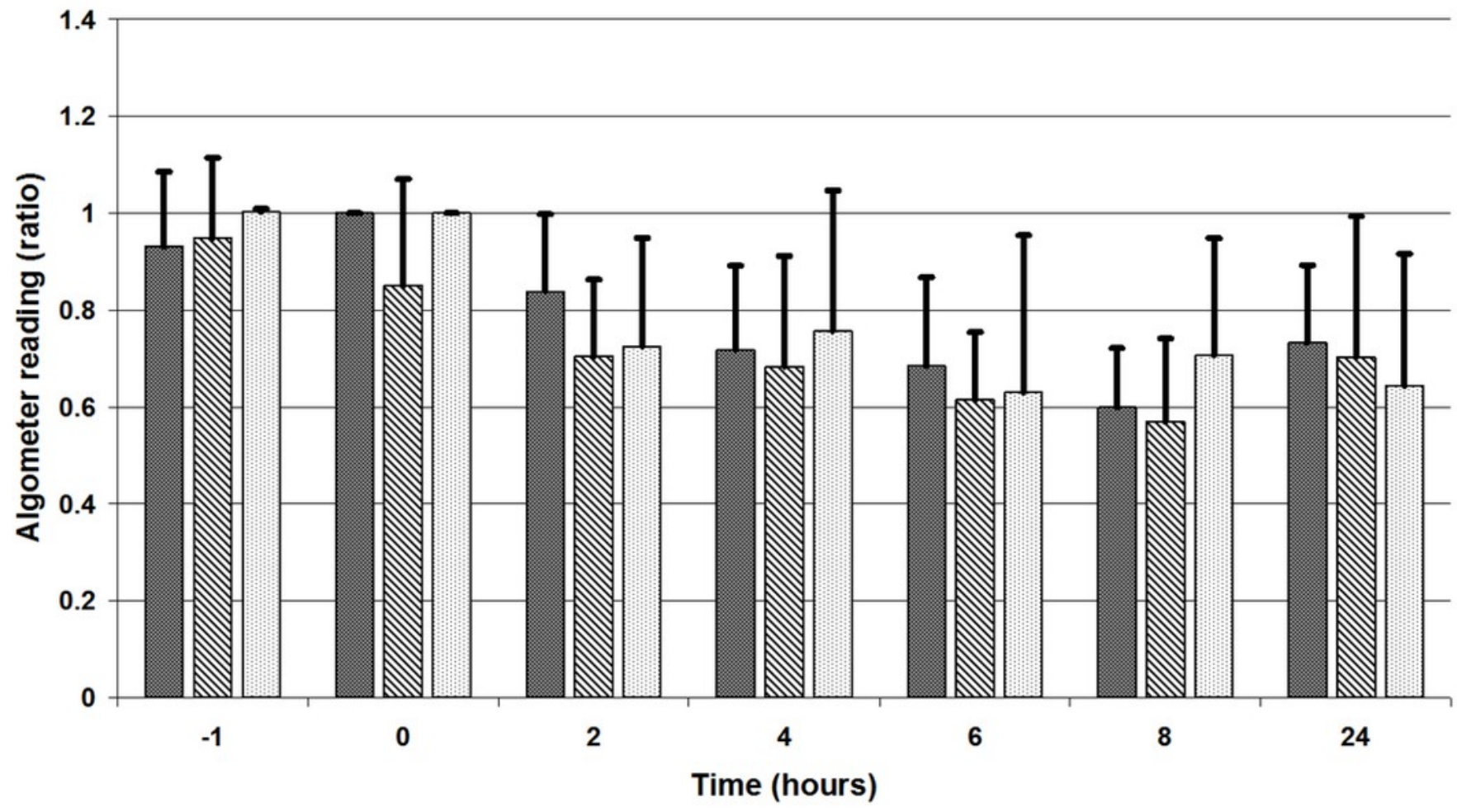




\section{Figure 5}

Figure 5

Algometric value, depicted as a ratio comparing the value obtained at each individual time point to values obtained at the abdomen prior to premedication (i.e. time, but not location, is the dependent variable). Time points for comparison to pre-medication values include premedication (time -1), extubation (time 0), and 2, 4, 6, 8 and 24 hours post-operatively. Error bars represent standard deviation. Error bars represent standard deviation. Note: L/B received saline premedication and local anesthetic line block, L/BM received morphine premedication and a local anesthetic line block, and SS received a saline premedication and saline line block. Also note that a ratio of one indicates the animal tolerates the same level of pressure on the abdomen at the time of comparison as it tolerated prior to incision. A decreasing ratio indicates the animal tolerates less pressure on the abdomen at the time of comparison as compared to pressure applied prior to the incision.

는 $\mathrm{B}$ $\mathbb{Q L L} / \mathrm{BM}$

TSS

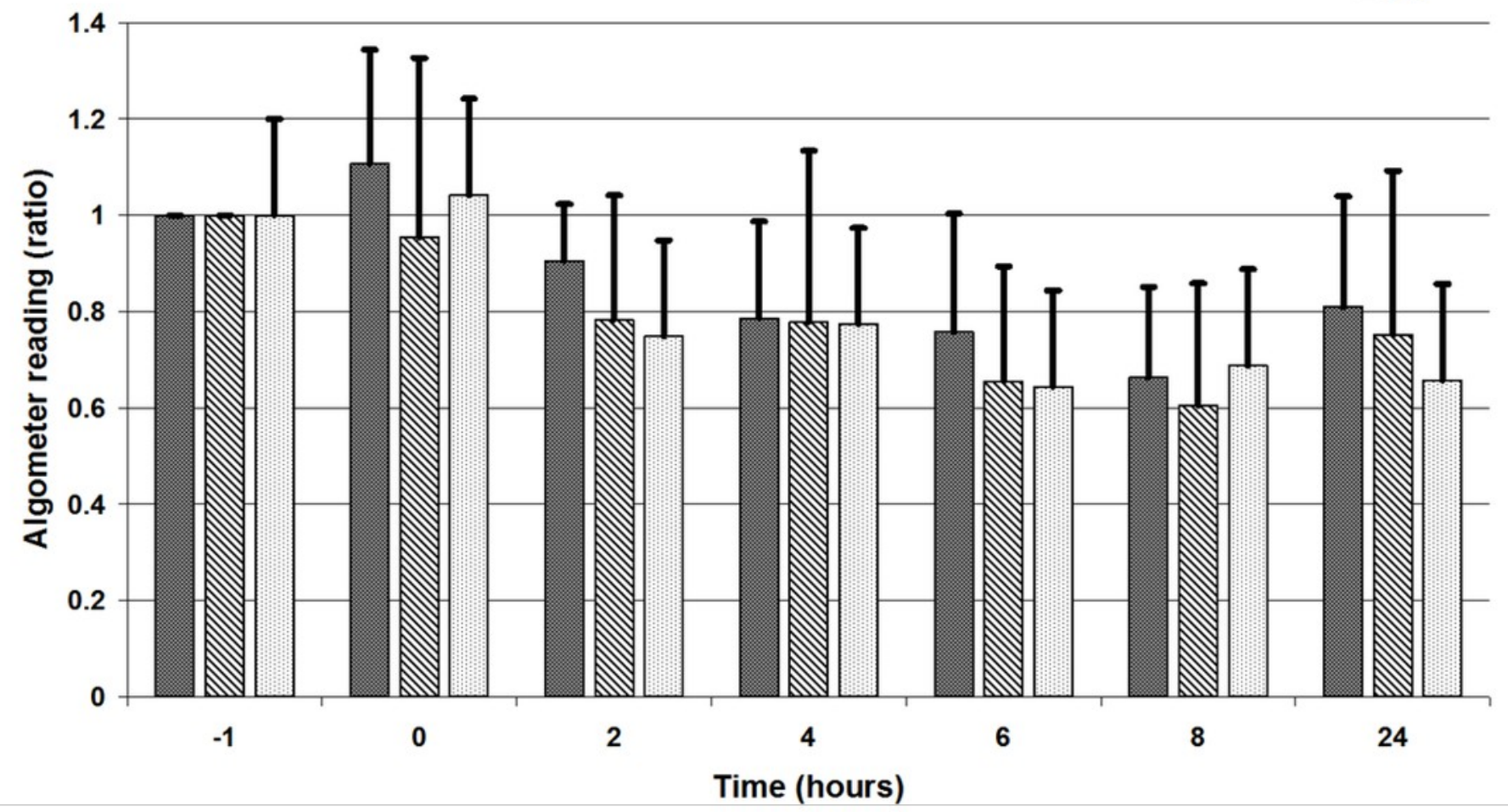




\section{Table 1 (on next page)}

\section{Table 1}

Baseline data for Groups L/B, L/BM, and SS. Data is presented as average ( $\pm S D)$, except for BUN, where average value only is listed. Respiratory rate was not included because a large number of animals were panting. 


\begin{tabular}{llll} 
Group & \multicolumn{1}{c}{ L/B } & \multicolumn{1}{c}{ L/BM } & SS \\
Number of dogs & 20 & 19 & 20 \\
Age (years) & $1.6 \pm 1.7$ & $1.6 \pm 1.4$ & $2.3 \pm 2.0$ \\
Weight (kg) & $17 \pm 6.8$ & $16.5 \pm 1.4$ & $18.2 \pm 9.6$ \\
Temperature (F) & $101.2 \pm 1.0$ & $101.1 \pm 0.9$ & $101.1 \pm 1.0$ \\
Heart rate (BPM) & $140 \pm 22$ & $138 \pm 26$ & $138 \pm 22$ \\
PCV $(\%)$ & $43 \pm 4.0$ & $42 \pm 4$ & $42 \pm 4$ \\
Total protein (g/dL) & $6.8 \pm 0.6$ & $6.8 \pm 0.7$ & $6.5 \pm 0.6$ \\
BUN (Azostick) & $5-15$ & $5-15$ & $5-15$ \\
Propofol (mg/kg) & $4.6 \pm 1.1$ & $4.3 \pm 1.6$ & $3.6 \pm 1.6$
\end{tabular}

